\title{
What is the relationship between body mass index and eating disorder symptomatology in female fashion models?
}

Christina Ralph-Nearman, $\mathrm{PhD}^{1,2^{*}}$, Hung-wen Yeh, $\mathrm{PhD}^{3}$, Sahib S. Khalsa, MD, $\mathrm{PhD}^{1,4}$, Jamie D. Feusner, $\mathrm{MD}^{5 * *}, \&$ Ruth Filik, $\mathrm{PhD}^{2 * *}$

${ }^{1}$ Laureate Institute for Brain Research (LIBR), Tulsa, Oklahoma, United States of America

${ }^{2}$ University of Nottingham, Nottingham, England

${ }^{3}$ Health Services \& Outcomes Research, Children's Mercy Hospital, Kansas City, Missouri, United States of America

${ }^{4}$ Oxley College of Health Sciences, University of Tulsa, Tulsa, Oklahoma, United States of America

${ }^{5}$ Semel Institute for Neuroscience and Human Behavior, University of California Los Angeles, United States of America

Running head: BMI AND EATING DISORDER SYMPTOMS: FASHION MODELS

*Corresponding Author

**Co-Last Authors

Christina Ralph-Nearman

Laureate Institute for Brain Research

6655 S Yale Avenue

Tulsa, OK 74133 United States

Email: ChristinaRalphNearman@gmail.com

Key words: body mass index, BMI, body image, eating disorders, fashion models 
BMI AND EATING DISORDER SYMPTOMS: FASHION MODELS

\begin{abstract}
Low body mass index $(\mathrm{BMI}<18 / 18.5)$ is utilized as a mandated cutoff for professional fashion model employment, based on assumptions that low BMI indicates eating disorder pathology. No previous studies have examined the association between experimenter-measured BMI and eating disorder symptomatology in professional fashion models. We measured BMI and Eating Disorder Examination Questionnaire (EDE-Q) responses in United Kingdom (UK) professional fashion models, and nonmodels. Characteristics were compared using robust standardized mean difference (rSMD) obtained via probability of superiority. Associations between BMI and eating disorder symptomatology were examined using robust regression, controlling for age. Models exhibited lower BMI but higher fat-percentage and muscle mass. On the EDE-Q, models had higher Restraint, Global, Eating, and Weight Concerns, and similar Shape Concern scores compared to nonmodels. BMI was positively associated with eating disorder symptoms in both groups, and all but one of the eight models with clinically significant EDE-Q level had $\geq 18.5$ measured BMI. Lower BMI was not indicative of worse eating disorder symptomatology in models or nonmodels. Thus, using a low BMI cutoff (<18.5) may not be an appropriate single index of health for detecting elevated eating disorder symptoms in models. Different policies to protect models' health should be considered.
\end{abstract}

Key words: body mass index; BMI; body image; eating disorders; fashion models; fashion; body image law 
BMI AND EATING DISORDER SYMPTOMS: FASHION MODELS

What is the relationship between body mass index and eating disorder symptomatology in female fashion models?

\section{Introduction}

Body Mass Index (BMI), which is calculated by dividing an individual's weight by the square of their height, was originally designed to classify average sedentary populations' weight health (World Health Organization, 1995). BMI is widely used for many purposes, such as a single health screening measure with school children, and an indicator of restored weight in individuals diagnosed with anorexia nervosa, despite lack of clarity regarding whether BMI is an appropriate single measure of an individual's health (Cook et al., 2005; Luke, 2009). In addition, for professional fashion models, many countries, such as France, Italy, Spain, and Israel, have mandated a minimum BMI cutoff (e.g., >18 or 18.5) to indicate 'fitness' to work. Other countries, such as the United States, United Kingdom (UK), Denmark, and Sweden are considering mandating a minimum BMI cutoff, as included in the 'Body Image Law', and recommended by experts for continued employment criteria (Bromberg \& Halliwell, 2016; Record \& Austin, 2016). That is, if a model is below a mandated BMI (e.g., $<18$ or $<18.5$ ), and thus deemed by these policy criteria as unhealthily thin, at risk for an eating disorder, and a sickly body image example for the public, then he/she may not legally work, for example, in fashion shows or photoshoots (Bromberg \& Halliwell, 2016; Record \& Austin, 2016).

\subsection{BMI cutoffs and the Body Image Law}

"The Body Image Law," which includes requiring professional fashion models to be above a minimum size and BMI, is suggested by Bromberg and Halliwell (2016) to be both 
BMI AND EATING DISORDER SYMPTOMS: FASHION MODELS

"Fashion Law" as it affects professional fashion models' ability to work, and also "Health Law," as it aims to improve public body image. While effects on the public are important and the main focus of many studies, it is also important to consider the health of professional fashion models. Although models are often considered to be a high-risk group for eating disorders, due to the industry criteria to be slim and tall (see e.g., Gladstone, 2016; Hoek, 2002), there is very limited research in this area, and no studies to date that provide objective evidence that professional fashion models' lower experimenter-measured BMI is associated with worse eating disorder symptoms. Most studies investigate the impact of 'skinny' models or media on the general population, which is also important (e.g., Halliwell \& Dittmar, 2004). However, there is no empirical evidence that an arbitrary BMI cutoff (e.g., $<18.5)$ is a valuable sole measure of eating disorder pathology in models, which is not surprising due to the complexity of this disorder. Furthermore, a BMI cut-off may misclassify individuals within the ‘healthy' BMI category ( $\geq 18.5-25)$ who have significant eating disorder symptoms, which would make BMI an inappropriate single measure to remove models from their employment.

The few studies that have investigated the relationship between models' self-reported BMI and their eating disorder symptomology have provided mixed findings. Studies have mainly relied on models' self-reported BMI, often collecting data by mail, or via online studies, instead of in-person experimenter-measured BMI of professional models in a prominent fashion city (e.g., London, Paris, New York, Milan)(Garner \& Garfinkel, 1980; Preti, Usai, Miotto, Pertretto, \& Masala, 2008; Rodgers, Ziff, Lowy, Yu, \& Austin, 2017; Van Hanswijck de Jonge \& Van Furth, 1999). While a good start of investigation, these are problematic designs for several reasons. Although self-reported BMI and measured BMI are highly correlated in 'overweight' or 
BMI AND EATING DISORDER SYMPTOMS: FASHION MODELS

'normal' weight samples (e.g., Gorber, Temblay, Mooher, \& Gorber, 2007), or BMI may be overestimated by individuals in a lower but 'healthy' BMI category $(<22)$ (Stommel \& Schoenborn, 2009), it is uncertain whether self-reported BMI is reliably associated with actual, measured-BMI in models, and whether experimenter-measured BMI is a valid sole measure of eating disorder severity in models. A prior study found that models were more accurate at estimating their body part measurements and overall size than nonmodels on a 3-dimensional avatar (Ralph-Nearman et al., 2019), which is expected, as models view their bodies often and are frequently measured for height and body areas to fit clothing. However, since models are not required to be a specific weight to fit into clothing, and not consistently weighed, it is uncertain if models may accurately know their own weight from day to day. The lack of importance of weight is demonstrated by a typical model's composite card (the business card a client will view to select a model for employment), which includes pertinent information for a client to hire the model for a photoshoot or runway show. That is the models' name, agency information, several pictures, eye and hair color, and measurements of their height, bust, waist, hips, dress, trousers, and shoe size.

\subsection{Studies related to models' self-reported BMI and eating disorder symptoms}

Earlier studies in which models and nonmodels self-reported their BMI, the models' reports indicated that they were significantly underweight (that is, self-reported BMI <18/18.5) (Brenner \& Cunningham, 1992; Preti et al., 2008; Santonastaso \& Favaro, 2002; Swami \& Szmigielska, 2013). Models perceived themselves as significantly taller than nonmodels but had similar eating disorder symptomatology (Brenner \& Cunningham, 1992; Preti et al., 2008; Santonastaso \& Favaro, 2002; Swami \& Szmigielska, 2013). Two studies conducted in Italy 
BMI AND EATING DISORDER SYMPTOMS: FASHION MODELS

found that models reported higher eating disorder symptomatology (on the EDE and EDE-Q) than nonmodels (Preti et al., 2008; Santonastaso \& Favaro, 2002).

Altogether, the evidence regarding whether BMI is related to weight control or eating disorder behaviors in fashion models is unclear, due to the lack of experimenter-measured BMI. Two studies with self-reported BMI (Preti et al., 2008; Santonastaso \& Favaro, 2002) also drew the conclusion that only a small number of professional fashion models met the criteria for an ED, or reported using unhealthy strategies to manipulate their weight. They suggested that fashion models were a high-risk group for "partial" ED, which would include one other criterion in addition to being underweight for anorexia nervosa or bingeing and/or purging for bulimia nervosa (such as their weight or shape dictating their self-worth). Finally, an online study recruiting models through social media reported that models perceived a great deal of pressure from their agencies to lose weight, and this perception was related to "unhealthy weight control behaviors" (Rodgers et al., 2017). Importantly, models themselves reported that they believed that imposing minimum BMI restrictions would be the least impactful method to protect models (Rodgers et al., 2017).

While some studies have reported that eating disorder behaviors may be the mechanism for models to attain and maintain a slim body (Preti et al., 2008; Santonastaso \& Favaro, 2002), another model-related study concluded that the slim body of professional female fashion models is perhaps often not caused by immoderate dieting behavior, but instead, best considered as, "lying at the extreme of the normal distribution of body types" (Brenner \& Cunningham, 1992).

The lack of studies collecting direct BMI measurements from professional models and the concurrent implementation of a weight-based public policy suggests that further research 
BMI AND EATING DISORDER SYMPTOMS: FASHION MODELS

involving objective physical measurements is needed (Zancu \& Enea, 2017).

\subsection{General population BMI and eating disorder relationship direction}

In general population studies, higher BMI has often been linked to greater levels of eating disorder tendencies, including restricting food intake. For instance, one study found that eating disorder symptoms, including restriction of food, increased with increasing BMI (Rø, Reas, \& Rosenbinge, 2012). Another two-year study that investigated the risk factors for binge eating onset indicated that higher BMI in adolescent girls predicted an extreme risk for binge eating (Stice, Presnell, \& Spangler, 2002). These findings raise an important question: Is there a relationship between experimenter-measured BMI and eating disorder symptoms in professional fashion models? And if so, in which direction? It is still unclear whether BMI is an appropriate indicator or "red flag" for eating disorder symptomatology in different populations.

\subsection{Current Study Predictions}

This is the first study to use experimenter-measured BMI in professional fashion models. We predicted that if models were similar to the general population, then higher BMI would be associated with more severe eating disorder symptoms. Similar to findings in general population studies, we predicted that in nonmodels, higher BMI would be associated with more severe eating disorder symptoms. We also explored the relationship between reported and experimentermeasured BMI in professional fashion models, due to the fact that no studies have investigated this association.

\section{Methods}

\subsection{Participants}


BMI AND EATING DISORDER SYMPTOMS: FASHION MODELS

Female professional models ( $n=67 ; 18-35$ years) were recruited through professional modeling agencies in the UK. Female nonmodels ( $n=218$; $18-37$ years) were recruited from the general population through flyers and social media in the UK and entered into a prize draw for their participation. The study was approved by the Psychology Department Ethics Review Board at the University of Nottingham.

\subsection{Procedure}

After giving written informed consent, each participant was seated at a computer and completed the Eating Disorder Examination Questionnaire (EDE-Q 6.0) (Fairburn \& Beglin, 2008). Fashion model participants were informed that their individual data would not be shared with their modeling agencies, and encouraged to answer as honestly as possible. This was followed by calculating each participant's BMI $\left(\mathrm{Kg} / \mathrm{M}^{2}\right)$ from their experimenter-measured height and weight, and using bioimpendance to measure fat-percentage and muscle mass (including skeletal and smooth muscles and the water within these muscles).

\subsection{Measures}

The EDE-Q 6.0 (Fairburn \& Beglin, 2008) is a 28-item validated and reliable questionnaire shown to be comparable to the clinical-rated tool (the EDE) (Berg, Peterson, Frazier, \& Crow, 2012). We chose this scale because it is the only measure included in a largescale health survey to assess the level and monitoring of eating disorder pathology (Health Survey For England (HSFE), 2017). The EDE-Q 6.0 provides frequency of eating disorder behaviors, as well as four facets of eating disorder symptomatology: Restraint Concern (five 
BMI AND EATING DISORDER SYMPTOMS: FASHION MODELS

items about avoiding eating), Eating Concern (five items about negative feelings associated with eating), Shape Concern (eight items about self-body shape concern), Weight Concern (five items about fear of gaining weight), and a global eating disorder score (the average of the sum of the four facets). A clinically significant eating disorder cutoff $(\geq 4.0)$ has been established by some researchers and clinicians, which was used in this current paper to point to severe eating disorder symptoms (Carter, Stewart, \& Fairburn, 2001; Luce, Crowther, \& Pole, 2008).

BMI $\left(\mathrm{Kg} / \mathrm{m}^{2}\right)$ guidelines are provided with the DSM-5 to index the 'significantly low weight' for a diagnosis of anorexia nervosa, which is: "Mild" > 17; "Moderate" between 16 and 16.99; "Severe" between 15 and 15.99; and "Extreme" < 15 (American Psychiatric Association, 2013). Experimenter-measured BMI and body composition (fat-percentage and muscle mass) were calculated for each participant, removing heavy clothing, shoes, and socks, by using a stadiometer for height and a bioimpedance scale for weight and body composition (Innerscan Segmental Body Composition Scale, Tanita, Inc.). Since most studies with models only utilize models' self-reported BMI, we additionally collected models' self-reported height and weight, in order to calculate models' self-reported BMI.

\subsection{Statistical analysis}

Categorical variables (eating disorder behaviors) were summarized by frequency and proportion, and continuous variables (group characteristics and EDE-Q scores) were summarized by mean (M) and standard deviation (SD) if the distributions were approximately Gaussian or by median and inter-quartile range (IQR) if otherwise, as the mean is sensitive to outliers. The internal consistency of EDE-Q items was assessed by Cronbach's $\alpha$. The between-group 
differences were evaluated by a unified standardized mean difference (SMD) for both categorical and continuous variables (Yang \& Dalton, 2012). However, it is well known that the conventional SMD is sensitive to assumptions of normality and equal variance (e.g., Li, 2016). In this work, we estimated between-group effect sizes using robust SMD (rSMD) via "probability of superiority" (Ruscio, 2008), a robust nonparametric estimator for the parametric common-language (CL) effect size (Cliff, 1993) (see Table 1 of Ruscio, 2008) so that Cohen's guidelines could still be applicable while the results were not sensitive to outliers or violation of Gaussian distributions. The between-group differences in continuous variables following and not following Gaussian distributions and categorical variables were examined using 2-sample $t$-, Wilcoxon-Mann-Whitney (WMW), and Chi-squared (with Yates' continuity corrections) tests, respectively. To explore the relationship between reported and experimenter-measured BMI in models, the within-group difference of self-reported vs.-measured BMI was assessed using Wilcoxon signed rank tests. We rely on Cohen's (1988) effect-sizes guidelines (Negligible difference: <.1; Small: .1 to .3; Medium: .3 to .5; and Large: .5 to 1.0), and so the resulting $p$ values were reported merely for readers' interests as no thresholds were applied (either raw $p$ values or controlled for multiple comparisons) for decision-making (see e.g. Wasserstein et al., 2019). To test if BMI would be associated with more severe eating disorder symptoms, and if so, in which direction, we examined associations between measured BMI and eating disorder symptomatology (EDE-Q scores), controlling for age, using robust regression and Huber estimation method, for each group of models and nonmodels and for both groups combined. We further explored the association for $\mathrm{BMI}<18.5$ and $\mathrm{BMI} \geq 18.5$ sub-groups within fashion models and within nonmodels, and calculated the frequency of models and nonmodels with 
clinically significant EDE-Q levels $(\geq 4.0)$ with measured BMI's above and below the proposed 18.0 and 18.5 cutoffs. The analyses were conducted in R statistical language using the psych package (Revelle, 2018) to compute Cronbach's $\alpha$, the tableone package (Yoshida, 2019) to create tables, the effsize package (Torchiano, 2018) to compute the conventional SMD (and author-developed R scripts to adjust SMD due to unequal sample sizes and compute robust SMD), and the MASS package (Venables and Ripley, 2002) to conduct robust regression.

\section{Results}

Following Cohen's (1988) guidelines, the fashion-models and nonmodels exhibited differences in age, percent fat, and muscle mass (robust SMD 0.12, 0.09, and 0.05, respectively, Table 1), difference in weight (SMD -0.03), height (robust SMD 0.19), and experimentermeasured BMI (robust SMD -0.66; the negative sign indicated lower BMI in models than nonmodels) (see overlapping histograms in Supplement Figure S.1.).

---- Table 1 goes about here ----

Regarding the eating disorder symptomatology scores on the EDE-Q 6.0 (Fairburn \& Beglin, 2008), the data suggested high internal consistency with Cronbach's $\alpha$ of 0.96 (95\% CI's, $0.95,0.97)$ for nonmodels, and 0.97 (95\% CI's, 0.96, 0.98) for fashion-models. The two groups exhibited some minor differences in the Restraint and Shape Concern (robust SMD 0.15 and 0.09; Table 1) in the raw scores but not in other scores (robust SMD 0 or 0.01). As the effect size between models and nonmodels for age was greater than small according to Cohen's (1988) 
guidelines ( $>0.10)$, age was considered a covariate. After regressing out age, the fashion models reported greater Restraint (robust SMD 0.21, $p=.01$ ) (Table 2), slightly greater Global EDE-Q, Eating and Weight Concern (rSMD 0.09, 0.14 and 0.07, $p=0.28,0.16$ and 0.31, respectively), and almost equal Shape Concern (rSMD $-0.02, p=0.72$ ) compared with nonmodels (the negative sign indicated less Shape Concern in models than nonmodels).

----Table 2 goes about here----

\subsection{EDE-Q Dietary restraint, binge eating, compensation, and purging frequency}

Within the prior 28 days, models and nonmodels reported very small differences between overall frequency of restraint behaviors ( $S M D s \leq 0.26$; $p s \geq 0.09$ ), binge eating behaviors $(S M D s \leq 0.26, p s \geq 0.10$ ), and frequency of compensation or purging behaviors ( $S M D s \leq 0.18$, $p s \geq 0.19)$. However, nonmodels showed higher frequency of binge eating with feelings of loss of control than models ( $\mathrm{SMD}=-0.35, p=0.02$ ), and models reported more laxative misuse than nonmodels ( $\mathrm{SMD}=0.37, p=0.02$ ) (see Table 3 ).

-----Table 3 goes about here ------

\subsection{Models' estimated vs. measured BMI Differences}

Fashion models' self-reported BMI $(M d n=17.0, I Q R=1.00)$ was lower than their experimenter-measured BMI ( $M d n=18.6, I Q R=1.25)$, with a median of difference -0.9 and IQR $0.80($ Wilcoxon signed rank statistic $=2123, p<.001)$. 


\subsection{Correlations between BMI and EDE-Q Scores}

In models, age-controlled experimenter-measured BMI was positively associated with global and all facets of EDE-Q scores (regression coefficients ranged from 0.30 and 0.63 , Table 4). This was similarly observed in nonmodels (regression coefficients ranged between 0.05 and 0.20) (see Figure 1). Interestingly, in a subsample of participants with BMI $\geq 18.5$, BMI was positively associated with the EDE-Q Global score in models (regression coefficient=0.70, $\mathrm{N}=37,95 \% \mathrm{CI}(0.06,1.34), p=0.03)$ and nonmodels (regression coefficient $=0.16, \mathrm{~N}=198$, 95\% CI $(0.07,0.26), p=0.001)$. However, in those with BMIs $<18.5$, this level of correlation was not seen in our subsample of models (regression coefficient $=0.05, \mathrm{~N}=30,95 \% \mathrm{CI}(-0.52$, $0.62), p=0.86$ ), or nonmodels (regression coefficient $=0.18, \mathrm{~N}=20,95 \% \mathrm{CI}(-0.18,0.54), p=$ $0.31)$.

Overall, there was only one participant (a model) who both exceeded the clinically significant cutoff on the EDE-Q $(\geq 4.0)$ and whose measured BMI was below the proposed 18.5 or 18.0 BMI cutoff $(\mathrm{BMI}=17.8$; EDE-Q=5.3). The other participants in both groups $(n=21)$ who met or exceeded a proposed threshold for clinicially significant eating disorder symptoms (EDE$\mathrm{Q} \geq 4.0$ ) were above the proposed 18.5 BMI cutoff: 14 nonmodels (BMI range=19.1-22.7 $(M=21.0, S D=1.3)$; EDE-Q range=4.1-5.5( $M=4.5, S D=0.4))$ and seven models $(\mathrm{BMI}$ range=18.6$21.2(M=19.7, S D=0.9))$; EDE-Q range=4.0-4.7 $(M=4.3, S D=0.3))$.

-----Figure 1 goes about here------

\subsection{Body composition correlations with EDE-Q scores}


Whereas muscle mass and eating disorder symptoms were inversely related, especially Global EDE-Q, Weight and Shape Concern in nonmodels, muscle mass and eating disorder symptoms were positively related in models (see Table 4). Higher fat percentage was associated with higher eating disorder symptoms in both groups (see Table 4).

----Table 4 goes about here----

\section{Discussion}

There are expert recommendations and policies being implemented using BMI as a regulated cutoff for the employment eligibility of professional models, under the assumption that models with a BMI below a certain threshold are likely to have an eating disorder and portray an 'unhealthy' body image to the public. To evaluate the relationship between BMI and eating disorder symptomatology we assessed experimenter-measured BMI and eating disorder symptoms (using the measure recommended in the UK (EDE-Q scores) (HSFE, 2017)) in a sample of female professional fashion models and a sample of female nonmodels in the UK. In the current study robust regressions provide evidence that higher experimenter-measured BMI is associated with higher levels of eating disorder symptomatology in professional fashion models.

\subsection{Discussion of results compared to prior studies}

In support of what some past studies have indicated within non-model populations (Fairburn \& Beglin, 2008; Kelly, Vimalakanthan, \& Miller, 2014; Rø et al., 2012; Stice et al., 2002), the current results suggest that BMI is positively related to eating disorder symptoms in both groups. Specifically, we found that higher measured BMI was related to higher levels of 
eating disorder symptomatology (EDE-Q) in both the nonmodel and in the professional fashion model samples. It has been proposed that the close resemblance of a model's body to cultural ideals may be a protective factor against negative body image in fashion models (Brenner \& Cunningham, 1992; Swami \& Szmigielska, 2013). As body image disturbance is a core feature of anorexia nervosa (APA, 2013), a more positive and less negative body image may in turn also protect models from eating disorder symptoms. In a similar manner, in several studies, female models have been reported to possess higher self-esteem (Brenner \& Cunningham, 1992), more positive body appreciation (Swami \& Szmigielska, 2013), and have comparable positive and negative body attitude to nonmodels (Brenner \& Cunningham, 1992; Preti et al., 2008; Swami \& Szmigielska, 2013). Consistent with another study with professional UK models ( $n=63)$ and controls ( $n=126)$ (Santonastaso \& Favro, 2002), professional models and nonmodels largely did not have statistically different eating disorder symptomatology or frequency of eating disorder behaviors (i.e., restraint, bingeing, purging, exercise compulsion, or compensation). When controlling for age in the current study, nonmodels reported more binge eating with feelings of loss of control than models, and models reported more laxative use than nonmodels. Although there was some frequency of restraint behavior differences between models and nonmodels when controlling for age in the current study, it is important to note that in our sample of models, mean Restraint was slightly higher, and our sample of nonmodels Restraint was slightly lower, than average Restraint reported in the largest normative sample (Mond et al., 2006). Our sample may have differed from this community sample because the citation is older (i.e., 2006), and it may be that the prior study does not reflect the current ED symptoms in the population. The positive robust regressions between BMI and EDE-Q global score in models (and nonmodels) implied 
that lower BMI was associated with lower eating disorder symptoms, a finding which is contrary to the professional fashion industry policy.

Interestingly, BMI was associated with eating disorder symptoms (EDE-Q Global score) in models and nonmodels with BMI in the 'normal' range $(\geq 18.5)$, but did not appear to be related in those with BMI considered 'underweight' $(<18.5)$. While the lack of such an association could be partly due to a smaller subsample for those with BMI $<18.5$, it provides initial evidence that BMI alone may not be an effective tool for inferring the presence of eating disorder symptoms. In fact, only one participant of the 285 studied displayed both clinically significant eating disorder symptoms $(\geq 4.0)$ and a BMI below the proposed 18.5 or 18.0 cutoffs. Instead, the 21 participants (14 nonmodels and seven models) with clinically significant eating disorder symptoms were all in the 'healthy' BMI range (18.6-22.7), underscoring the utility of a symptom-based measurement.

\subsection{Implications of the results}

Together, these results suggest that establishing a low BMI cutoff $(<18.5)$ as a primary indicator that a model has an eating disorder may not be appropriate, as lower BMI was associated with lower eating disorder symptoms. These results seem to relate to other studies which point to a percentage of healthy adults within the "underweight" BMI category $(<18.5)$ in the general population (WHO, 1996), and findings that weight-restored ( $\geq 18.5 \mathrm{BMI}$ ) individuals diagnosed with anorexia nervosa may continue to exhibit every other criterion for the disorder (Zhang et al., 2016). Importantly, if a low BMI cutoff $(<18.5)$ was used to infer the presence of an eating disorder in models, results from this present study suggest that those models within what is considered a 'normal' or 'healthy' BMI range $(\geq 18.5$ and $<25)$ with significant eating 
disorder symptoms ( $\geq 4.0$ on the EDE-Q) might be overlooked. It may be that the slim body of professional female fashion models may be, as previously described, "lying at the extreme of the normal distribution of body types.” (Brenner \& Cunningham, 1992).

It is important to consider that if lower measured BMI is not significantly associated with higher eating disorder symptoms for professional fashion models, as found in our present study, then removing models from their employment using a BMI cutoff $(<18.5)$ may be unfounded. Tantleff-Dunn et al. (2009) reported that even when an observer is informed that an underweight woman in a picture may have her body type due to heredity, she may be at-risk for being stereotyped as unhealthy (with possible implications that she is undereating and depressed), because of her body size. Therefore, requiring a $<18.5$ BMI cutoff of individuals who do not present eating disorder symptoms may send a message to the public that all thin bodies $(<18.5$ BMI) are unhealthy bodies, which is the flip-side of the false message that all larger bodies (>25 BMI) are unhealthy bodies. In their book “Women Can’t Win”, Bruner, Valine, and Ceja (2016) suggest that "Women are in a Catch-22 situation, trapped between fat-shaming and skinnyshaming" (page 244).

The findings of this study suggest that it is important to question the utility of BMI monitoring as a sole indicator of an eating disorder in professional fashion models. If fashion industry regulations require using a BMI cutoff, this could lead to models being weighed more regularly, which could then potentially exacerbate eating disorder symptoms. In fact, there is evidence that continuous weight monitoring may inadvertently increase eating disorder risk. For instance, a longitudinal study of children found that regularly enforced weight monitoring predicted greater levels of eating disorder tendencies (frequency of binge eating, fasting, 
vomiting, laxative use, diet pills, etc.) in the adolescent female stage (Neumark-Sztainer et al., 2007). Additionally, a review of evidence about the effect of self-weighing on psychological well-being concluded that self-weighing may be psychologically harmful for younger women attentive to dieting (see Pacanowski et al., 2016). Thus, it has been proposed that the regular judgements related to weight, shape, and food that fashion models may be subjected to as part of the 'professional development' of this career may actually elevate their eating disorder risk (Hoek, 2002; Treasure \& Roberts, 2008). Thus, enforcing regular, ongoing weight and BMI monitoring in young fashion models may lead to long term negative health effects.

\subsection{Models' reported and experimenter-measured BMI comparison}

Our exploration of the relationship between models' self-reported versus experimentermeasured BMI revealed that, while both groups spanned the "underweight" and "healthy weight" BMI categories, both the professional fashion models' and nonmodels' average measured BMIs were within the 'healthy weight' range (18.6 and 21.4 respectively). However, professional models perceived their BMI (i.e., 17.0 on average) to be significantly lower $(p<.001)$ than their experimenter-measured BMI (i.e., 18.6 on average), with a large effect size. The average BMI value reported by our model samples is similar to other studies with models' self-reported BMI, perhaps suggesting that models also perceived themselves to be lower in weight than they actually were in the previous studies that relied upon self-reported BMI (Brenner \& Cunningham; Santonastaso \& Favaro, 2002). Individuals in the 'healthy' and 'overweight' BMI category often underestimate their body size, which is theorized to be due to them calibrating their body perception with a general population that is progressively becoming more overweight. 
BMI AND EATING DISORDER SYMPTOMS: FASHION MODELS

This may be related to the self-serving bias theory (Mazzurega et al., 2018). Interestingly, Mazzurega et al. reported that greater self-serving bias was related to less eating disorder risk. As models significantly underestimated their body weight (median difference $0.9, p<.001$ ) in relation to their actual BMI in our present study, this may indicate body-related self-serving bias.

\subsection{Models' and nonmodels' body fat and muscle composition comparison}

Models' experimenter-measured level of fat and muscle mass were both slightly higher than nonmodels' in our samples. Models' higher muscle mass level was associated with more severe eating disorder symptoms. Nonmodels displayed the opposite pattern of effects, with lower muscle mass associated with higher eating disorder symptoms. Whilst models' higher measured muscle mass was positively associated with eating disorder symptoms, nonmodels' decreased measured muscle mass was associated with increased eating disorder symptoms. That is, models with greater levels of muscle mass reported higher eating disorder behaviors, whilst nonmodels with less muscle mass reported greater eating disorder behaviors.

There may be a number of reasons that greater muscle mass was related to higher risk for eating disorder symptoms for models. For instance, models who are weight training and intentionally building more muscle mass may appear to have more restraint eating behaviors, e.g. if they were participating in certain dieting practices such as 'intermittent fasting.' Alternatively, it may be that models who are naturally more muscular may want to try to slim down and do this by food restraint. Or perhaps eating disorder behaviors in some of the models with higher symptoms include exercising more, which could result in building more muscle from working out. Nevertheless, the current research indicates that future measurements of BMI and the 
relationship to eating disorder symptoms might benefit from assessments of body composition, dieting practices (e.g., restriction of food and misuse of laxatives), and more detailed examination of attitudes toward food.

\subsection{Limitations and future directions}

Although this is the first study to directly measure professional fashion models' BMI, body composition, and eating disorder symptomatology, it is important to note certain limitations and considerations for future investigations. Due to time constraints, we did not utilize clinician interviews to assess eating disorder symptomatology in models or nonmodels, which has been proposed as an in-depth means of evaluating the presence of eating psychopathology, unless individuals are uncomfortable to verbally disclosing the severity of the issue (Smolak \& Levine, 2018). Instead, we used the EDE-Q, which is a validated and reliable questionnaire that can provide a reasonable approximation of eating disorder symptoms, shown to be comparable to the clinical evaluation tool (the EDE) (Berg et al., 2012). It is also important to note that, as has been found in other studies (Ralph-Nearman \& Filik, 2018), the EDE-Q scores also differed slightly from those from another community sample of young females from Australia (Mond et al., 2006). This is perhaps not unusual, as eating disorder symptomatology may vary across cultures (Mond et al., 2006; Tozzi et al., 2005). Also, we did not obtain an estimated BMI from nonmodels, and therefore cannot compare these results between groups. Not a limitation per se, but it is also important to note that although in both our model and nonmodel samples the median BMI was within the 'healthy' range, this is much lower than the average female in the UK, which is typically overweight or obese (HSFE, 2017). However, the similarity in weight between 
groups may make them better comparisons for this study. Another limitation of this study is that it is cross-sectional and employed correlational analyses; thus, cause and effect relationships between eating disorder symptom severity and BMI cannot be established. If a direct relationship exists, it is plausible that higher BMI could contribute to eating disorder behaviors or that eating disorder behaviors could contribute to higher BMI. Perhaps more likely is that there are subsets of individuals (models and nonmodels) with different relationships between BMI and eating disorder symptoms; our subanalyses of those with $\geq 18.5$ and $<18.5$ suggests that this might be the case, although our sample size is too low to draw definitive conclusions.

\subsection{Conclusions}

In conclusion, while BMI is a readily measurable metric, it is neither sensitive nor specific enough as a sole measure to detect an eating disorder such as anorexia nervosa in professional fashion models, and may neglect individuals within the 'healthy' BMI range $(\geq 18.5)$ who are above the clinical ED threshold (EDE-Q $\geq 4.0$ ). This is unsurprising given the complexity of psychiatric conditions. The current finding that models have lower BMIs but similar levels of overall eating disorder symptoms compared to nonmodels challenge the notion that solitary measurement of BMI can serve as an objective indicator of an eating disorder in models. At the same time, the observation that models reported using laxatives more frequently suggests that the inclusion of symptom and behavior assessment may provide additional insight into the factors that are relevant to the expression of an eating disorder. While safeguards do need to be put in place for professional fashion models' own health and also as a 'healthy' portrayal to the public, perhaps a better way of assessing this and protecting the health and 
BMI AND EATING DISORDER SYMPTOMS: FASHION MODELS

livelihood of fashion models needs to be developed and considered regarding policy. This could include, for the public, mandating that designers produce a wider range in sample clothing sizes to be inclusive of a range of body sizes for their runways and advertisments. It has been proposed that models, similar to professional athletes, may need strategies unique from interventions effective with nonmodels to assist healthy body image (Swami \& Szmigielska, 2013). This suggestion may be extended to include less invasive and implicit detection of eating disorder symptoms, and immediate referral to a clinician when needed, as well ensuring that there is access to appropriate treatment and support for high-risk models. 
BMI AND EATING DISORDER SYMPTOMS: FASHION MODELS

\section{Statements}

\section{Acknowledgement}

The authors would like to thank the professional modelling agencies and research participants for their contributions.

\section{Disclosure Statement}

The authors have no conflicts of interest to declare.

\section{Data Availability Statement}

The data are not shared due to privacy or ethical restrictions.

\section{Funding Sources}

The authors would like to acknowledge the funding support from the University of Nottingham's Vice-Chancellor's Scholarship for Research Excellence (C.R.N.). 


\section{References}

American Psychiatric Association. (2013). Diagnostic and statistical manual of mental disorders (DSM-5®). Arlington, VA: American Psychiatric Publication. doi.org/10.1176/appi.books.9780890425596

Berg, K. C., Peterson, C. B., Frazier, P., \& Crow, S. J. (2012). Psychometric evaluation of the eating disorder examination and eating disorder examination-questionnaire: A systematic review of the literature. International Journal of Eating Disorders, 45(3), 428-438.

Bromberg, M., \& Halliwell, C. (2016). All about That Bass and Photoshopping a Model's Waist: Introducing Body Image Law. The University of Notre Dame Australia Law Review, 18,1 .

Brenner, J.B., \& Cunningham, J. G. (1992). Gender differences in eating attitudes, body concept, and self-esteem among models. Sex Roles, 27(7-8), 413-437. doi.org/10.1007/BF00289949

Bruner, M. S., Valine, K., \& Ceja, B. (2016). Women Can't Win. Politics, Protest, and Empowerment in Digital Spaces, 244.

Canty, A., \& Ripley, B. (2017). boot: Bootstrap R (S-Plus) Functions. R package version 1.3-20.

Carter, J. C., Stewart, D. A., \& Fairburn, C. G. (2001). Eating disorder examination questionnaire: norms for young adolescent girls. Behaviour Research and Therapy, 39(5), $625-632$.

Cliff, N. (1993). Dominance statistics: Ordinal analyses to answer ordinal questions. Psychological Bulletin, 114, 494-509. doi.org/10.1037/0033-2909.114.3.494

Cohen J. (1988). Statistical Power Analysis for the Behavioral Sciences. Routledge. 
BMI AND EATING DISORDER SYMPTOMS: FASHION MODELS

Cook, Z., Kirk, S., Lawrenson, S., \& Sandford, S. (2005). Use of BMI in the assessment of undernutrition in older subjects: reflecting on practice. Proceedings of the Nutrition Society, 64(3), 313-317. doi.org/10.1079/PNS2005437

Garner, D.M. \& Garfinkel, P.E. (1980). Socio-cultural factors in the development of anorexia nervosa. Psychological Medicine, 10, 647-656.

doi.org/10.1017/S0033291700054945

Gladstone, J. (2016). The skinny on BMI-based hiring: an assessment of the legality and effectiveness of Israel's weight restriction law. Wash. U. Global Stud. L. Rev., 15, 495.

Gorber, S. C., Tremblay, M., Moher, D., \& Gorber, B. (2007). A comparison of direct vs. selfreport measures for assessing height, weight and body mass index: a systematic review. Obesity reviews, 8(4), 307-326. doi.org/10.1111/j.1467-789X.2007.00347.x

Fairburn, C.G., \& Beglin, S. J. (2008). Cognitive Behavior Therapy and Eating Disorders. Eating Disorder Examination Questionnaire (6.0). ed. F. CG. New York: Guilford Press.

Halliwell, E., \& Dittmar, H. (2004). Does size matter? The impact of model's body size on women's body-focused anxiety and advertising effectiveness. Journal of Social and Clinical Psychology, 23(1), 104-122.

Health Survey for England 2017 (HSFE). (2017). Overweight and obesity in adults and children. [cited 2020 April 7]; Available from: http://healthsurvey.hscic.gov.uk.

Hoek, H.W. (2002). Distribution of eating disorders. Eating disorders and obesity: A Comprehensive Handbook, 2, 233-237. 
Kelly, A.C., Vimalakanthan, K., \& Miller, K. E. (2014). Self-compassion moderates the relationship between body mass index and both eating disorder pathology and body image flexibility. Body Image, 11(4), 446-453. doi.org/10.1016/j.bodyim.2014.07.005

Kim, S. (2015). ppcor: Partial and Semi-Partial (Part) Correlation. R package version 1.1. https://CRAN.R-project.org/package=ppcor

Li, J. C. H. (2016). Effect size measures in a two-independent-samples case with nonnormal and nonhomogeneous data. Behavior Research Methods, 48,1560- 1574. doi.org/10.3758/s13428-015-0667-z

Luce, K. H., Crowther, J. H., \& Pole, M. (2008). Eating Disorder Examination Questionnaire (EDE-Q): Norms for undergraduate women. International Journal of Eating Disorders, 41(3), 273-276.

Luke, A. (2009). Ethnicity and the BMI-body fat relationship. British Journal of Nutrition, 102(4), 485-487. doi.org/10.1017/S0007114508207233

Mazzurega, M., Marisa, J., Zampini, M., \& Pavani, F. (2018). Thinner than yourself: self-serving bias in body size estimation. Psychological Research, 1-18.

Mond, J.M., Hay, P. J., Rodgers, B., \& Owen, C. (2006). Eating Disorder Examination Questionnaire (EDE-Q): Norms for young adult women. Behaviour Research and Therapy, 44(1), 53-62. doi.org/10.1016/j.brat.2004.12.003

Neumark-Sztainer, D.R., Wall, M. M., Haines, J. I., Story, M. T., Sherwood, N. E., \& Van den Berg, P. A. (2007). Shared risk and protective factors for overweight and 
disordered eating in adolescents. American Journal of Preventive Medicine, 33(5), 359369. doi.org/10.1016/j.amepre.2007.07.031

Pacanowski, C. R., Linde, J. A., \& Neumark-Sztainer, D. (2015). Self-weighing: Helpful or harmful for psychological well-being? A review of the literature. Current Obesity Reports, 4(1), 65-72.

Preti, A., Usai, A., Miotto, P., Petretto, D. R., \& Masala, C. (2008). Eating disorders among professional fashion models. Psychiatry Research, 159(1-2), 86-94. doi.org/10.1016/j.psychres.2005.07.040

Ralph-Nearman, C., Arevian, A. C., Puhl, M., Kumar, R., Villaroman, D., Suthana, N., ...\& Khalsa, S. S. (2019). A Novel mobile tool (Somatomap) to assess body image perception pilot tested with fashion models and nonmodels: Cross-sectional study. JMIR Mental Health, 6(10), e14115. doi:10.2196/14115

Ralph-Nearman, C., \& Filik, R. (2018). Eating disorder symptomatology and body mass index are associated with readers' expectations about character behavior: Evidence from eyetracking during reading. International Journal of Eating Disorders, 51(9), 1079-1079. doi.org/10.1002/eat.22961

Record, K.L., \& Austin, S. B. (2016). "Paris Thin": A Call to Regulate Life-Threatening Starvation of Runway Models in the US Fashion Industry. American Journal of Public Health, 106(2), 205-206. doi.org/10.2105/AJPH.2015.302950

Revelle, W. (2018). psych: Procedures for Personality and Psychological Research, Northwestern University, Evanston, Illinois, USA, https://CRAN.Rproject.org/package $=$ psych Version $=1.8 .12$. 
Rø, Ø., Reas, D. L., \& Rosenvinge, J. (2012). The impact of age and BMI on Eating Disorder Examination Questionnaire (EDE-Q) scores in a community sample. Eating Behaviors, 13(2), 158-161. doi.org/10.1016/j.eatbeh.2011.12.001

Rodgers, R.F., Ziff, S., Lowy, A. S., Yu, K., \& Austin, S. B. (2017). Results of a strategic science study to inform policies targeting extreme thinness standards in the fashion industry. International Journal of Eating Disorders, 50(3), 284-292. doi:10.1002/eat.22682

Ruscio, J. (2008). A probability-based measure of effect size: Robustness to base rates and other factors. Psychological Methods, 13, 19-30. doi.org/10.1037/1082-989X.13.1.19

Santonastaso P, M.S., \& Favaro A. (2002). Are fashion models a group at risk for eating disorders and substance abuse? Psychotherapy Psychosomatics, 71, 168-172. doi.org/10.1159/000056285

Smolak, L., \& Levine, M. P. (2018). Diagnostic Interview. Eating Disorders: Understanding Causes, Controversies, and Treatment [2 volumes], 166.

Stice, E., Presnell, K., \& Spangler, D. (2002). Risk factors for binge eating onset in adolescent girls: a 2-year prospective investigation. Health Psychology, 21(2), 131. doi.org/10.1037//0278-6133.21.2.131

Stommel, M., \& Schoenborn, C. A. (2009). Accuracy and usefulness of BMI measures based on self-reported weight and height: findings from the NHANES \& NHIS 2001-2006. BMC Public Health, 9(1), 421. 
Swami, V., \& Szmigielska, E. (2013). Body image concerns in professional fashion models: Are they really an at-risk group? Psychiatry Research, 207(1-2), 113-117. doi.org/10.1016/j.psychres.2012.09.009

Tantleff-Dunn, S., Hayes, S., \& Braun, C. P. (2009). How did you get so thin? The effect of attribution on perceptions of underweight females. Eating and Weight Disorders-Studies on Anorexia, Bulimia and Obesity, 14(1), 38-44.

Torchiano, M. (2018). effsize: Efficient Effect Size Computation. doi: 10.5281/zenodo.1480624 (URL: http://doi.org/10.5281/zenodo.1480624), R package version 0.7.4, <URL: https://CRAN.R-project.org/package=effsize〉.

Treasure J. L., W. E., \& Roberts M. E. (2008). Models as a high-risk group: the health implications of a size zero culture. British Journal of Psychiatry, 192(4), 243-244. doi.org/10.1192/bjp.bp.107.044164

Tozzi, F., Thorton, L. M., Klump, K. L., Fichter, M. M., Halmi, K. A., Kaplan, A. S., ... Rotondo, A. (2005). Symptom fluctuation in eating disorders: Correlates of diagnostic crossover. American Journal of Psychiatry, 162(4), 732-740. doi.org/10.1176/appi.ajp.162.4.732

Van Hanswijck de Jonge, P., \& Van Furth, E. (1999). Eating disorders in models: fiction or fact? European Eating Disorders Review, 7, 235-238. Doi.org/10.1002/(SICI)1099-0968(199908)7:4<235::AIDERV297>3.0.CO:2-E

Venables, W. N. \& Ripley, B. D. (2002) Modern Applied Statistics with S. Fourth Edition. Springer, New York. ISBN 0-387-95457-0 
Wasserstein, R.L., Schirm, A.L., \& Lazar, N.A. (2019). Moving to a World Beyond “p < 0.05.” The American Statistician, 73, 1-19.

World Health Organization. (1995). Physical status: The use of and interpretation of anthropometry. Report of a WHO Expert Committee. Retrieved on March 20, 2020: https://apps.who.int/iris/bitstream/handle/10665/37003/WHO_TRS_854.pdf

Yang, D., \& Dalton, JE. (2012). A unified approach to measuring the effect size between two groups using SAS. SAS Global Forum 2012, Paper, 335-2012.

Yoshida, K. (2019). tableone: Create 'Table 1' to Describe Baseline Characteristics. R package version 0.10.0. https://CRAN.R-project.org/package=tableone

Zancu, S. A., \& Enea, V. (2017). Eating disorders among fashion models: a systematic review of the literature. Eating and Weight Disorders-Studies on Anorexia, Bulimia and Obesity, 22(3), 395-405. doi.org/10.1007/s40519-016-0293-5

Zhang, A., Leow, A., Zhan, L., GadElkarim, J., Moody, T., Khalsa, S., ... \& Feusner, J. D. (2016). Brain connectome modularity in weight-restored anorexia nervosa and body dysmorphic disorder. Psychological Medicine, 46(13), 2785-2797. doi.org/10.1017/S0033291716001458 
BMI AND EATING DISORDER SYMPTOMS: FASHION MODELS

\section{Figures}

\section{Figure 1}

Robust regression between Global EDE-Q scores and measured BMI for fashion models $(n=67)$ and nonmodels $(n=218)$.

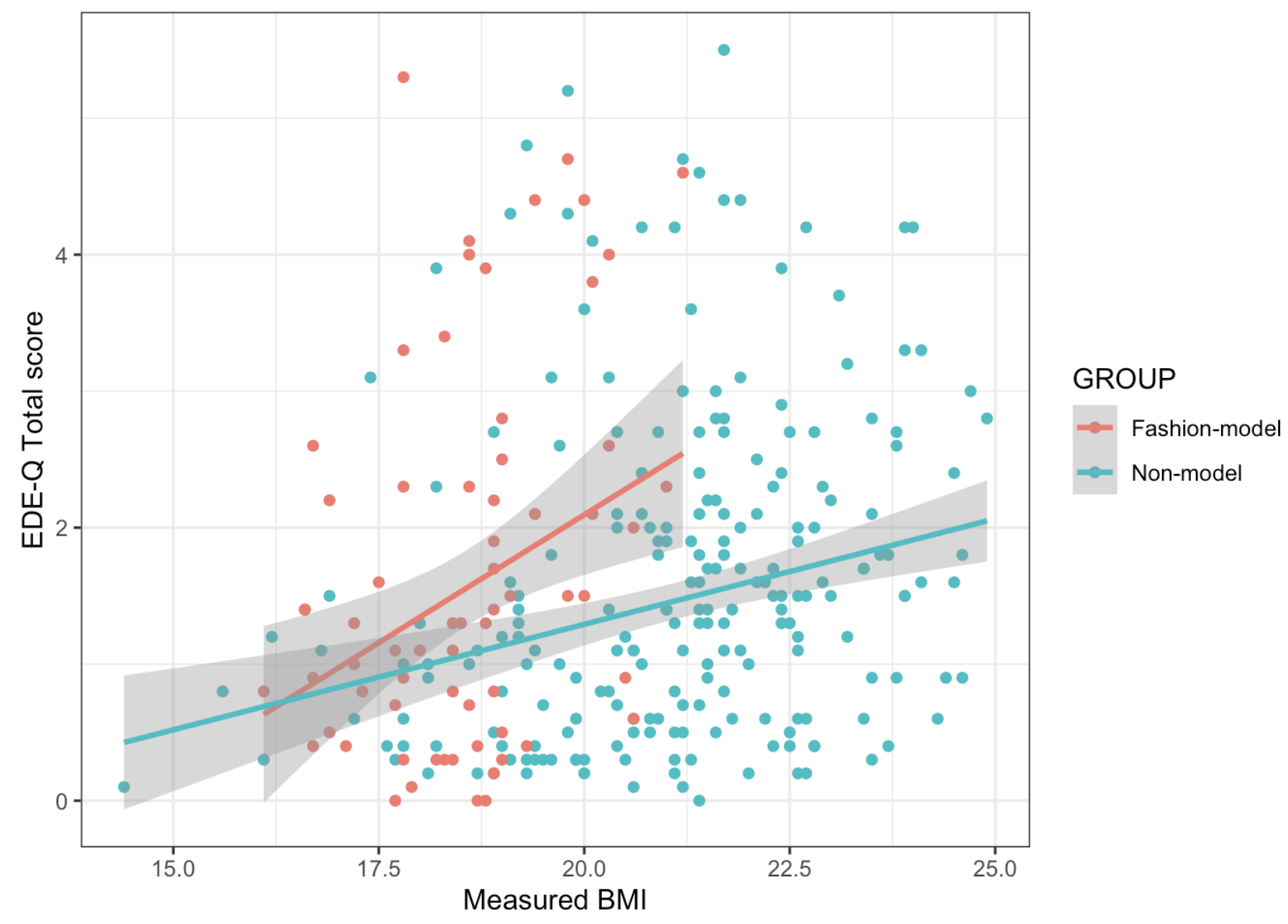


Table 1

Tables

Differences in characteristics and EDE-Q scores between fashion models and nonmodels.

\begin{tabular}{|c|c|c|c|c|}
\hline Variable & Models $(N=67)$ & Nonmodels $(N=218)$ & $p$-value & $\mathrm{rSMD} \dagger$ \\
\hline Age $(y r s)^{a}$ & $23[20,25]$ & $21[19,23]$ & $<0.001$ & 0.12 \\
\hline Weight (lbs) ${ }^{b}$ & $127.1(9.5)$ & $127.5(15.7)$ & 0.84 & 0 \\
\hline Height $(\mathrm{cm})^{\mathrm{a}}$ & $177.0[172,179]$ & $165.1[161.0,170.2]$ & $<0.001$ & 0.19 \\
\hline $\mathrm{BMI}^{\mathrm{a}}$ & $18.6[17.8,19.1]$ & $21.4[19.9,22.4]$ & $<0.001$ & -0.66 \\
\hline Fat $(\%)^{\mathrm{a}}$ & $23.7[20.8,26.3]$ & $20.5[17.8,22.8]$ & $<0.001$ & 0.09 \\
\hline Muscle Mass (lbs) ${ }^{\mathrm{a}}$ & $91.4[87.1,97.2]$ & $87.7[84.6,90.8]$ & $<0.001$ & 0.05 \\
\hline Global EDE-Q $^{a}$ & $1.30[0.65,2.30]$ & $1.40[0.60,2.20]$ & 0.99 & 0 \\
\hline Restraint $^{\mathrm{a}}$ & $1.20[0.60,2.60]$ & $0.80[0.20,1.80]$ & 0.04 & 0.15 \\
\hline Eating Concern ${ }^{\mathrm{a}}$ & $0.40[0.20,1.40]$ & $0.60[0.20,1.20]$ & 0.90 & 0.01 \\
\hline Weight Concern ${ }^{\mathrm{a}}$ & $1.80[0.60,3.50]$ & $1.60[0.80,2.80]$ & 0.97 & 0 \\
\hline Shape Concern ${ }^{\mathrm{a}}$ & $1.90[0.80,3.05]$ & $2.10[1.02,3.50]$ & 0.17 & -0.09 \\
\hline
\end{tabular}

Note. ${ }^{\mathrm{a}}$ Median [IQR] and the Wilcoxon-Mann-Whitney test; ${ }^{\mathrm{b}}$ Mean (SD) and 2-sample $t$-test; $\dagger$ robust SMD obtained via probability of superiority; yrs=years; lbs=pounds; $\mathrm{cm}=$ centimeters 
BMI AND EATING DISORDER SYMPTOMS: FASHION MODELS

\section{Table 2}

Between-group differences in EQE-Q scores controlling for age.

\begin{tabular}{l|c|c|c|c} 
Variable & Models $(N=67)$ & Nonmodels $(N=218)$ & $p$ value & rSMD $\dagger$ \\
\hline Global EDE-Q $^{\mathrm{a}}$ & $-0.29[-0.82,0.89]$ & $-0.32[-0.90,0.50]$ & 0.28 & 0.09 \\
Restraint $^{\mathrm{a}}$ & $-0.05[-0.80,1.32]$ & $-0.59[-1.08,0.50]$ & 0.01 & 0.21 \\
Eating Concern $^{\mathrm{a}}$ & $-0.28[-0.68,0.67]$ & $-0.33[-0.70,0.28]$ & 0.16 & 0.14 \\
Weight Concern $^{\mathrm{a}}$ & $-0.04[-1.27,1.71]$ & $-0.33[-1.16,0.88]$ & 0.31 & 0.07 \\
Shape Concern $^{\mathrm{a}}$ & $-0.33[-1.24,0.88]$ & $-0.22[-1.14,1.08]$ & 0.72 & -0.02 \\
\hline
\end{tabular}

${ }^{a}$ Median [IQR] of residuals (regressed out age) and the Wilcoxon-Mann-Whitney test; $\dagger$ robust SMD obtained via probability of superiority 
BMI AND EATING DISORDER SYMPTOMS: FASHION MODELS

\section{Table 3}

Distributions of eating disorder behaviors within the prior 28 days

Compared between fashion models' and nonmodels.

\begin{tabular}{|c|c|c|c|c|}
\hline Variable & Models $(\mathrm{N}=67)$ & Nonmodels $(\mathrm{N}=218)$ & $p$ value & SMD \\
\hline Restraint $\geq 8$ hours $^{\text {a }}$ & $19(28.4)$ & $39(17.9)$ & 0.09 & 0.26 \\
\hline Restraint $\geq 13$ days $^{\mathrm{a}}$ & $3(4.5)$ & $8(3.7)$ & 0.72 & 0.04 \\
\hline Binge $^{a}$ & $43(64.2)$ & $160(73.4)$ & 0.19 & -0.2 \\
\hline Binge $\geq 4$ times $^{\mathrm{a}}$ & $20(29.9)$ & $42(19.3)$ & 0.10 & 0.26 \\
\hline Binge + Loss control ${ }^{\mathrm{a}}$ & $28(41.8)$ & $129(59.2)$ & 0.02 & -0.35 \\
\hline $\begin{array}{l}\text { Binge+Loss control } \\
\geq 4 \text { times }\end{array}$ & $22(32.8)$ & $56(25.7)$ & 0.32 & 0.16 \\
\hline $\begin{array}{l}\text { Binge+Loss control } \\
\geq 13 \text { days }\end{array}$ & $5(7.5)$ & $10(4.6)$ & 0.36 & 0.13 \\
\hline Compulsive exercise & $32(47.8)$ & $93(42.7)$ & 0.55 & 0.1 \\
\hline $\begin{array}{l}\text { Compulsive exercise } \\
\geq 20 \text { times }\end{array}$ & $5(7.5)$ & $8(3.7)$ & 0.19 & 0.18 \\
\hline $\begin{array}{l}\text { Self-induced } \\
\text { vomiting }\end{array}$ & $4(6.0)$ & $9(4.1)$ & 0.51 & 0.09 \\
\hline Laxative misuse & $7(10.4)$ & $6(2.8)$ & 0.02 & 0.37 \\
\hline
\end{tabular}

${ }^{\mathrm{a}}$ Frequency count (\%) and the Wilcoxon-Mann-Whitney test 
BMI AND EATING DISORDER SYMPTOMS: FASHION MODELS

Table 4

Robust regressions and 95\% CI between BMI, body compositions and EDE-Q 6.0 scores

Fashion models

Nonmodels

\begin{tabular}{c|c|c|c|c|c|c|c|c|c} 
& Indep. & Coef. & & & $p$ & Coef. & & & $p$ \\
Response & Var. & Est. & lo & hi & & Est. & lo & hi & \\
\hline BMI & Global & 0.48 & 0.08 & 0.88 & 0.02 & 0.16 & 0.07 & 0.25 & $<0.001$ \\
BMI & Restraint & 0.51 & 0.10 & 0.93 & 0.02 & 0.15 & 0.06 & 0.24 & 0.002 \\
BMI & EC & 0.30 & 0.02 & 0.58 & 0.04 & 0.05 & -0.01 & 0.11 & 0.114 \\
BMI & WC & 0.50 & -0.02 & 1.01 & 0.06 & 0.21 & 0.09 & 0.33 & 0.001 \\
BMI & SC & 0.63 & 0.10 & 1.16 & 0.02 & 0.20 & 0.06 & 0.34 & 0.005 \\
Fat (\%) & Global & 0.00 & -0.10 & 0.11 & 0.93 & -0.01 & -0.03 & 0.02 & 0.72 \\
Fat (\%) & Restraint & -0.01 & -0.12 & 0.10 & 0.83 & -0.02 & -0.05 & 0.01 & 0.22 \\
Fat (\%) & EC & -0.01 & -0.08 & 0.07 & 0.85 & -0.00 & -0.02 & 0.01 & 0.70 \\
Fat (\%) & WC & 0.03 & -0.10 & 0.17 & 0.62 & 0.00 & -0.03 & 0.04 & 0.81 \\
Fat (\%) & SC & -0.01 & -0.15 & 0.13 & 0.88 & -0.00 & -0.05 & 0.04 & 0.84 \\
Muscle (lbs) & Global & 0.02 & -0.06 & 0.09 & 0.66 & -0.01 & -0.02 & -0.00 & 0.005 \\
Muscle (lbs) & Restraint & 0.03 & -0.05 & 0.10 & 0.50 & -0.01 & -0.02 & -0.00 & 0.04 \\
Muscle (lbs) & EC & 0.01 & -0.04 & 0.06 & 0.65 & -0.01 & -0.01 & -0.00 & 0.02 \\
Muscle (lbs) & WC & 0.03 & -0.07 & 0.12 & 0.56 & -0.02 & -0.03 & -0.00 & 0.008 \\
Muscle (lbs) & SC & -0.00 & -0.10 & 0.09 & 0.98 & -0.02 & -0.03 & -0.01 & 0.005 \\
\hline
\end{tabular}

Note. Indep. Var. = Independent Variable; Coef. Est.= Coeffient estimates; EC=Eating concern; $\mathrm{WC}=$ Weight concern; $\mathrm{SC}=$ Shape concern 


\section{Supplement}

\section{Figure S.1.}

Distributions of experimenter-measured weight, height, BMI, fat, and muscle mass for fashion models and nonmodels.
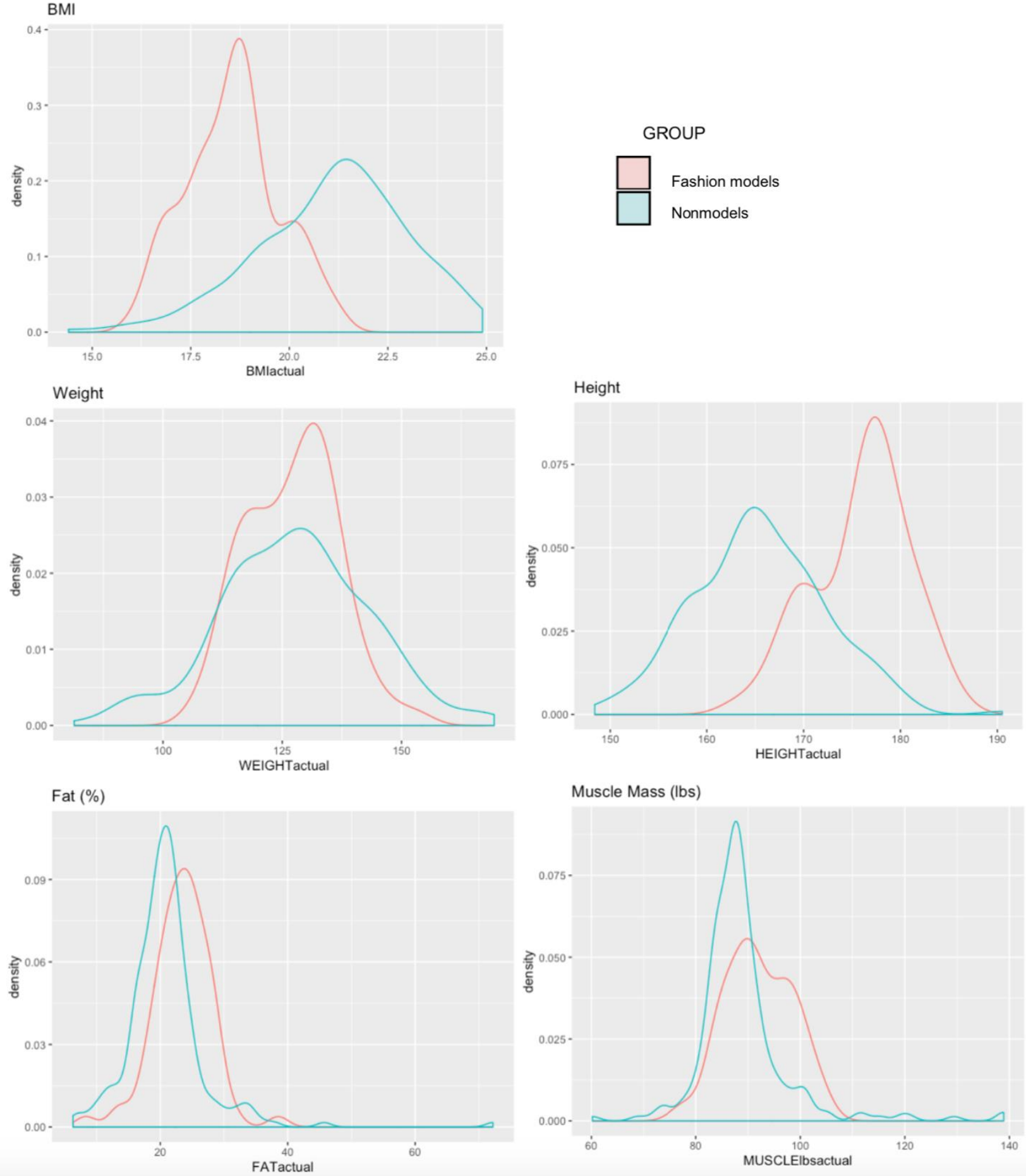